J. Appl. Numer. Optim. 1 (2019), No. 2, pp. 183-199

Available online at http://jano.biemdas.com

https://doi.org/10.23952/jano.1.2019.2.08

\title{
GENERALIZED MONOTONE MIXED EQUILIBRIUM PROBLEMS IN FC-SPACES: EXISTENCE AND APPROXIMATION
}

\author{
GAYATRI PANY ${ }^{1}$, OUAYL CHADLI ${ }^{2}$, RAM N. MOHAPATRA ${ }^{3, *}$ \\ ${ }^{1}$ Engineering Systems and Design Pillar, Singapore University of Technology and Design, Singapore \\ ${ }^{2}$ Department of Economics, Ibn Zohr University, B.P. 8658 Poste Dakhla, Agadir, Morocco \\ ${ }^{3}$ Department of Mathematics, University of Central Florida, USA
}

\begin{abstract}
In this paper, we study a class of mixed equilibrium problems under the extended generalized $\alpha-\eta$ monotonicity in finitely continuous topological spaces. Existence of solutions to the problems is established by relaxing the convexity structure and the linearity condition by using the relatively Knaster-Kuratowski-Mazurkiewicz principle. We also propose an iterative scheme based on auxiliary principle techniques. The results obtained in this paper improve and generalize some recent results in the framework of FC spaces under weaker conditions. They are useful to solve problems, where the domain and range of the underlying mappings lack the convexity structure.
\end{abstract}

Keywords. Auxiliary principle technique; FC-space; Generalized $\alpha-\eta$ monotonicity; Mixed equilibrium problem; R-KKM principle.

2010 Mathematics Subject Classification. 47H04, 47H10, 46N10

\section{INTRODUCTION}

Equilibrium problems are considered as the unifying concept that embodies various interesting and important mathematical models, such as, optimization problems, game theory, variational inequalities and complementarity problems. They were primarily introduced by Blum and Oettli [4]. Recently, they have been extensively studied by many authors in the context of both Hilbert and Banach spaces under different kinds of generalized monotonicities, such as, pseudomonotonicity, quasimonotonicity, relaxed monotonicity and semimonotonicity. Generally the underlying functions in most of the equilibrium and variational inequality problems involved in real life applications are not monotone. This fact motivates many researchers to extend the concept of the monotonicity to more weaker form. Weaker versions of the monotonicity like semimonotonicity and relaxed monotonicity have been studied by many authors. Bai, Zhou and Ni [2] studied variational-like inequalities, under the relaxed $\eta-\alpha$ pseudomonotonicity. Recently, Chen and Luo [5] introduced the relaxed $\eta-\alpha$ quasimonotone and the relaxed $\eta-\alpha$ proper quasimonotone mappings to study generalized variational-like inequality problems. In all these results, the underlying convexity assumptions appear to be strict from the viewpoint of applications. Sometimes it is encountered that in the study of some real life problems the domain and range of mappings may

\footnotetext{
${ }^{*}$ Corresponding author.

E-mail addresses: gp11@iitbbs.ac.in (G. Pany), o.chadli@uiz.ac.ma (O. Chadli), ram.mohapatra@ucf.edu (R.N. Mohapatra).

Received June 27, 2019; Accepted August 5, 2019.
}

(C)2019 Journal of Applied and Numerical Optimization 
lack the convexity structure. Such problems fit the framework of finitely continuous topological spaces (in short FC-spaces) without any convexity and linear structure. This class of spaces was introduced by Ding $[6,7]$ for generalized games and problems in mathematical economics.

The aim of this paper is to study mixed equilibrium problems under the extended generalized $\alpha$ monotonicity in FC-spaces with both qualitative and approximation approaches via an iterative scheme with the aid of auxiliary principle techniques. We also discuss the existence of solutions under the generalized $\alpha$-pseudomonotonicity.

Let $E$ be a finitely continuous topological space and let $K$ be a finitely continuous subset of $E$. We consider the following extended generalized mixed equilibrium problem: Find $\bar{x} \in K$ such that

$$
\phi(y, \bar{x}, z)+b(\bar{x}, z)-b(\bar{x}, \bar{x}) \geq 0, \text { for all } y, z \in K,
$$

where $\phi: K \times K \rightarrow \mathbb{R}$ is a trifunction and $b: E \times E \rightarrow \mathbb{R}$ is a bifunction.

Recently, the extended generalized mixed equilibrium problem has been studied by Pany-MohapatraPani [13] when $K$ is an FC-subspace of a reflexive Banach space $E$. It involves particularly the problem studied in FC-spaces by Wang [18]. If $b \equiv 0$ and $\phi(y, x, z)=\psi(x, \eta(z, x))$, where $\eta: K \times K \rightarrow E$ is a mapping, then problem (1.1) is reduced to the following problem studied by Liu-Zeng in [12]:

Find $\bar{x} \in K$ such that $\psi(\bar{x}, \eta(z, \bar{x})) \geq 0$, for all $z \in K$.

The following nonlinear mixed variational-like inequality problem

$$
\text { find } \bar{x} \in K \text { such that }\langle N(\bar{x}, y), \eta(z, \bar{x})\rangle+b(\bar{x}, z)-b(\bar{x}, \bar{x}) \geq 0, \forall y, z \in K
$$

studied in $[13,14]$, can be obtained as a special case of (1.1) if $\phi(y, x, z)=\langle N(x, y), \eta(z, x)\rangle$, where $N: E \times E \rightarrow E^{*}$ and $\eta: K \times K \rightarrow E$. Here, $E$ denotes a topological vector space with its dual space $E^{*}$ and $K$ is an FC-subspace of $E$.

If $\phi(y, x, z)=\psi(x, z)$ and $b \equiv 0$, then (1.1) is reduced to the following classical equilibrium problem, introduced by Blum and Oettli [4]

$$
\text { find } \bar{x} \in K \text { such that } \psi(\bar{x}, z) \geq 0 \text {, for all } z \in K \text {. }
$$

The results obtained in this paper are new and improve recent existence results in literatures on equilibrium problems and mixed variational inequalities in FC-spaces. The paper is organized as the following. In Section 2, we give some basic notions and preliminary results. In Section 3, existence of solutions for extended generalized mixed equilibrium problem (1.1) are established by relaxing the convexity condition and the linearity structure of the considered space and using the R-KKM (relatively Knaster-Kuratowski-Mazurkiewicz) principle. The problem is studied under the relaxed $\alpha$-monotonicity and the relaxed $\alpha$-pseudomonotonicity assumptions. Section 4 is devoted to the existence of solutions to mixed variational-like inequalities in FC-spaces as applications of the main results obtained in Section 3. In Section 5, by means of the auxiliary principle techniques, we give an approximation of solutions to problem (1.1), where $K$ is an FC-subspace of $E$. We exhibit an algorithm which generates a sequence converging strongly to a solution of problem (1.1), and give an application to the approximation of solutions of mixed variational-like inequalities in FC-spaces. A conclusion on the study of solutions of equilibrium problems and mixed variational inequalities in FC-spaces is given in the last section, Section 6. 


\section{PRELIMINARIES}

Let $X$ be a topological space. We denote by $2^{X}$ and $\langle X\rangle$ the family of all subsets of $X$ and the family of all nonempty finite subsets of $X$, respectively. For a subset $A$ of $X$, we denote by $c l_{X}(A)$ the closure of $A$ in $X$. Let $\triangle_{n}$ be the standard $n$-dimensional simplex with vertices $e_{0}, e_{1}, \cdots, e_{n}$. If $J=\left\{i_{0}, i_{1}, \cdots, i_{k}\right\}$ is a nonempty subset of $\{0,1, \cdots, n\}$, we denote by $\triangle_{k}=\operatorname{co}\left(\left\{e_{j}: j=0, \cdots, k\right\}\right)$ the convex hull of the vertices $\left\{e_{j}: j \in J\right\}$. For any given subset $A$ of $X$, the compact closure of $A$, denoted by $\operatorname{ccl}(A)$, is defined as

$$
\operatorname{ccl}(A)=\cap\{B \subset X: A \subset B \text { and } B \text { is compactly closed in } X\} .
$$

Note that $A$ is compactly closed if and only if $\operatorname{ccl}(A)=A$.

Definition 2.1. Let $Y$ be a nonempty set and $K$ be a nonempty closed subset of a topological space $X$. A set-valued mapping $T: Y \rightarrow 2^{K}$ is said to be transfer closed-valued if, for any $(y, x) \in Y \times K$ with $y \notin T(x)$, there exists $y^{\prime} \in Y$ such that $x \notin c l_{K}\left(T\left(y^{\prime}\right)\right)$.

Remark 2.1. (i) It is easy to see that if $T: Y \rightarrow 2^{K}$ is closed-valued, then it is transfer closed-valued.

(ii) If $T: Y \rightarrow 2^{K}$ is transfer closed-valued, then it is transfer compactly closed-valued. For the definition of transfer compactly closed-valued operators, we refer to Ding [8, Definition 3.1].

Definition 2.2. [8] Let $Y$ be a nonempty set. Let $K$ be a nonempty closed subset of a topological space $X$ and let $\psi: Y \times K \rightarrow \mathbb{R}$ be a bifunction. Then, we say that $\psi$ is transfer lower (respectively, upper) semicontinuous in the second variable if, for each $\lambda \in \mathbb{R}$ and $(y, x) \in Y \times K$ with $\psi(y, x)>\lambda$ (respectively, $\psi(y, x)<\lambda)$, there exists $y^{\prime} \in Y$ and a neighbourhood $N(x)$ of $x$ in $K$ such that $\psi\left(y^{\prime}, z\right)>\lambda$ (respectively, $\left.\psi\left(y^{\prime}, z\right)<\lambda\right)$ for all $z \in N(x)$.

Remark 2.2. Let $T: Y \rightarrow 2^{K}$ be a set-valued mapping defined by

$$
T(y)=\{x \in K: \psi(y, x) \leq \lambda\}, \quad(\text { respectively, } T(y)=\{x \in K: \psi(y, x) \geq \lambda\})
$$

One can easily verify that $T$ is transfer closed-valued if and only if $\psi(y, x)$ is transfer lower (respectively, upper) semicontinuous in $x$.

Proposition 2.1. [17] Let $X$ and $Y$ be two topological spaces, and let $T: X \rightarrow 2^{Y}$ be a set-valued mapping. Then, $T$ is a transfer closed-valued mapping if and only if

$$
\bigcap_{x \in X} T(x)=\bigcap_{x \in X} \operatorname{cl}(T(x)) .
$$

The following notion of a finitely continuous topological space (in short, FC-space) was introduced by Ding in [6].

Definition 2.3. $\left(E,\left\{\phi_{N}\right\}\right)$ is said to be an FC-space if $E$ is a topological space and for each $N=$ $\left\{x_{0}, x_{1}, \cdots, x_{n}\right\} \in\langle X\rangle$, there exists a continuous mapping $\phi_{N}: \triangle_{n} \rightarrow E$. A subset $K$ of $\left(E,\left\{\phi_{N}\right\}\right)$ is said to be an FC-subspace of $E$ if, for each $N=\left\{x_{0}, x_{1}, \cdots, x_{n}\right\} \in\langle X\rangle$ and for each $\left\{x_{i_{0}}, x_{i_{1}}, \cdots, x_{i_{k}}\right\} \subset K \cap N$, $\phi_{N}\left(\triangle_{k}\right) \subset K$, where $\triangle_{k}=c o\left(\left\{e_{j}: j=0, \cdots, k\right\}\right)$.

FC-spaces contain $L$-convex spaces [3], $H$-spaces [10] and $G$-convex spaces [15]. The following example illustrates the concept of FC-space when the convex property fails for some sets. 
Example 2.1. Let $C_{1}$ and $C_{2}$ be two nonempty convex sets in a Banach space $E$ with $\operatorname{cl}\left(C_{1}\right) \cap \operatorname{cl}\left(C_{2}\right)=\emptyset$. Then $C=C_{1} \cup C_{2}$ is not convex. Let $l: C_{2} \rightarrow C_{1}$ be a mapping. For $N=\left\{x_{0}, x_{1}, \cdots, x_{n}\right\} \in\langle C\rangle$, let us set $N_{1}=\left\{x_{0}, x_{1}, \cdots, x_{j}\right\} \subset C_{1}$ and $N_{2}=\left\{x_{j+1}, \cdots, x_{n}\right\} \subset C_{2}$. Define a mapping $\phi_{N}: \triangle_{n} \rightarrow E$ by

$$
\phi_{N}\left(t_{0}, t_{1}, \cdots, t_{n}\right)= \begin{cases}\sum_{i=0}^{n} t_{i} x_{i}, & \text { if } N \subset C_{1} \text { or } N \subset C_{2}, \\ \sum_{i=0}^{j} t_{i} x_{i}+\sum_{i=j+1}^{n} t_{i} l\left(x_{i}\right), & \text { if } N=N_{1} \cup N_{2} .\end{cases}
$$

One can easily verify that $\phi_{N}$ is continuous, hence $C$ is an FC-space.

Definition 2.4. Let $X$ be a nonempty set and $\left(E,\left\{\phi_{N}\right\}\right)$ be an FC-space. A set-valued mapping $T: X \rightarrow$ $2^{E}$ is said to be a generalized $R$-KKM mapping (or that the family of sets $\{T(x)\}_{x \in X}$ has the $R$-KKM property) if, for each $\left\{x_{0}, x_{1}, \cdots, x_{n}\right\} \in\langle X\rangle$, there exists $N=\left\{y_{0}, y_{1}, \cdots, y_{n}\right\} \in\langle E\rangle$ such that for each subset $\left\{i_{0}, i_{1}, \cdots, i_{k}\right\} \subset\{0,1, \cdots, n\}, \phi_{N}\left(\triangle_{k}\right) \subset \cup_{j=0}^{k} T\left(x_{i_{j}}\right)$.

Theorem 2.1. [9] Let $X$ be a nonempty set and let $\left(E,\left\{\phi_{N}\right\}\right)$ be an FC-space. Let $T: X \rightarrow 2^{E}$ be a transfer compactly closed-valued set-valued mapping. Suppose that $T$ is a generalized $R$-KKM mapping and that for some $M \in\langle X\rangle, \cap_{x \in M} \operatorname{ccl}(T(x))$ is compact. Then, $\cap_{x \in X} T(x) \neq \emptyset$.

Definition 2.5. Let $K$ be an FC-subspace of $\left(E,\left\{\phi_{N}\right\}\right)$. A trifunction $\phi: K \times K \times K \rightarrow \mathbb{R}$ is said to be

(i) monotone if and only if $\phi(y, w, v)+\phi(y, v, w) \leq 0$, for all $y, v, w \in K$;

(ii) weakly relaxed $\alpha$-monotone if and only if there exists $\alpha: E \times E \rightarrow \mathbb{R}$ such that

$$
\phi(y, x, z)+\phi(y, z, x) \leq \alpha(x, z), \text { for all } x, y, z \in K
$$

(iii) weakly relaxed $\alpha$-pseudomonotone if and only if there exists $\alpha: E \times E \rightarrow \mathbb{R}$ such that

$$
\phi(y, x, z) \geq 0 \Longrightarrow \phi(y, z, x) \leq \alpha(x, z), \text { for all } x, y, z \in K
$$

(iv) $\gamma$-strongly monotone (here $E$ is supposed to be a normed space) if and only if there exists a positive constant $\gamma$, such that,

$$
\phi(y, x, z)+\phi(y, z, x) \leq-\gamma\|x-z\|^{2}, \text { for all } x, y, z \in K .
$$

Definition 2.6. Let $K$ be an FC-subspace of $\left(E,\left\{\phi_{N}\right\}\right)$. A bifunction $b: K \times K \rightarrow \mathbb{R}$ is said to be

(i) skew-symmetric if and only if

$$
b(x, z)+b(z, x)-b(x, x)-b(z, z) \leq 0, \text { for all } x, z \in K .
$$

(ii) $\gamma$-strongly skew-symmetric (here $E$ is supposed to be a normed space) if and only if there exists a positive constant $\gamma$, such that,

$$
b(x, z)+b(z, x)-b(x, x)-b(z, z) \leq-\gamma\|x-z\|^{2}, \text { for all } x, z \in K .
$$

Remark 2.3. Skew-symmetric bifunctions have certain properties, see [1], which can be regarded as analogs of the conditions governing the gradient monotonicity and nonnegativity of the second derivative of convex functions.

Definition 2.7. Let $K$ be an FC-subspace of $\left(E,\left\{\phi_{N}\right\}\right)$. 
(i) A function $f: K \rightarrow \mathbb{R}$ is said to be $R$-convex if, for each $N=\left\{x_{0}, x_{1}, \cdots, x_{n}\right\} \in\langle K\rangle$,

$$
f\left(x_{t}\right) \leq \sum_{i=0}^{n} t_{i} f\left(x_{i}\right)
$$

where $x_{t}=\phi_{N}\left(\sum_{i=0}^{n} t_{i} e_{i}\right), t_{i} \in[0,1], \sum_{i=0}^{n} t_{i}=1$.

(ii) A trifunction $\phi: K \times K \times K \rightarrow \mathbb{R}$ is said to be $R$-upper hemicontinuous if, for any $N=\left\{x_{0}, x_{1}\right\} \in$ $\langle K\rangle$ and $y, z \in K$,

$$
\limsup _{t \rightarrow 0^{+}} \phi\left(y, x_{t}, z\right) \leq \phi\left(y, x_{0}, z\right),
$$

where $x_{t}=\phi_{N}\left((1-t) e_{0}+t e_{1}\right), t \in[0,1]$.

Definition 2.8. Let $K$ be an FC-subspace of $\left(E,\left\{\phi_{N}\right\}\right)$, where $E$ is a topological vector space with dual $E^{*}$. If $T: K \rightarrow E^{*}$ and $\eta: K \times K \rightarrow E$, then $T$ is said to be $(\eta, R)$-upper hemicontinuous if for for any $N=\left\{x_{0}, x_{1}\right\} \in\langle K\rangle$ and $z \in K$,

$$
\limsup _{t \rightarrow 0^{+}}\left\langle T\left(x_{t}\right), \eta\left(z, x_{t}\right)\right\rangle \leq\left\langle T\left(x_{0}\right), \eta\left(z, x_{0}\right)\right\rangle,
$$

where $x_{t}=\phi_{N}\left((1-t) e_{0}+t e_{1}\right), t \in[0,1]$.

Definition 2.9. Let $K$ be an FC-subspace of $\left(E,\left\{\phi_{N}\right\}\right)$. A trifunction $\phi: K \times K \times K \rightarrow \mathbb{R}$ is said to be $\lambda$-generalized $R$-diagonally convex if for each finite subsets $\left\{y_{0}, \cdots, y_{n}\right\}$ and $N=\left\{x_{0}, \cdots, x_{n}\right\}$ of $K$, and any $x_{t}=\phi_{N}\left(\sum_{i=0}^{n} t_{i} e_{i}\right)\left(t_{i} \geq 0, \sum_{i=1}^{n} t_{i}=1\right)$, we have

$$
\sum_{i=0}^{n} t_{i} \phi\left(y_{i}, x_{t}, x_{i}\right) \geq 0
$$

Definition 2.10. Let $K$ be an FC-subspace of $\left(E,\left\{\phi_{N}\right\}\right)$, where $E$ is a topological vector space with dual space $E^{*}$. Let $N: K \times K \rightarrow E^{*}$ be a nonlinear operator and let $\eta: E \times E \rightarrow E$ be a vector-valued bifunction. The operator $N$ is said to be $(\eta, \lambda)$-generalized $R$-diagonally convex if for each finite subsets $\left\{y_{0}, \cdots, y_{n}\right\}$ and $N=\left\{x_{0}, \cdots, x_{n}\right\}$ of $K$, and any $x_{t}=\phi_{N}\left(\sum_{i=0}^{n} t_{i} e_{i}\right)\left(t_{i} \geq 0, \sum_{i=1}^{n} t_{i}=1\right)$, we have $\sum_{i=1}^{n} t_{i}\left\langle N\left(x_{t}, y_{i}\right), \eta\left(x_{i}, x_{t}\right)\right\rangle \geq$ $\lambda$.

Definition 2.11. Let $K$ be an FC-subspace of $\left(E,\left\{\phi_{N}\right\}\right)$, where $E$ is a topological vector space with dual space $E^{*}$. Let $N: E \times E \rightarrow E^{*}$ be a nonlinear operator and let $\eta: E \times E \rightarrow E$. The operator $N$ is said to be

(i) $\eta$-monotone if and only if $\langle N(x, y)-N(z, y), \eta(x, z)\rangle \geq 0$ for all $x, y, z \in K$;

(ii) weakly relaxed $(\eta, \alpha)$-monotone if and only if there exists $\alpha: E \times E \rightarrow \mathbb{R}$ such that

$$
\langle N(x, y)-N(z, y), \eta(x, z)\rangle \geq \alpha(z, x) \text { for all } x, y, z \in K .
$$

Remark 2.4. Let $N: E \times E \rightarrow E^{*}$ be a nonlinear operator and $\eta: E \times E \rightarrow E$ such that $\eta(w, v)=$ $-\eta(v, w)$. If $N$ is weakly relaxed $(\eta,-\alpha)$-monotone, then the trifunction $\phi: E \times E \times E \rightarrow \mathbb{R}$ defined by $\phi(y, w, v)=\langle N(w, y), \eta(v, w)\rangle$ is weakly relaxed $\alpha$-monotone.

Definition 2.12. Let $K$ be an FC-subspace of $\left(E,\left\{\phi_{N}\right\}\right)$, where $E$ is a topological vector space with dual $E^{*}$. If $T: K \rightarrow E^{*}$ and $\eta: K \times K \rightarrow E$, then $T$ is said to be

(i) $\delta$-strongly positive if and only if there exists $\delta>0$ such that $\langle T(w), w\rangle \geq \delta\|w\|^{2}$, for all $w \in E$; 
(ii) monotone if and only if $\langle T(w)-T(v), w-v\rangle \geq 0$, for all $w, v \in K$;

(iii) $\gamma$-strongly monotone if and only if there exists $\gamma>0$ such that $\langle T(w)-T(v), w-v\rangle \geq \gamma\|w-v\|^{2}$, for all $w, v \in K$.

Remark 2.5. If $T: E \rightarrow E^{*}$ is linear, bounded and $\delta$-strongly positive, then it is $\delta$-strongly monotone and $\|T\|$-Lipschitz continuous, i.e. $\|T(w)-T(v)\| \leq\|T\|\|w-v\|$ where $\|T\|$ is the norm of $T$.

\section{EXISTENCE RESULTS}

In this section, we give some existence results for the mixed equilibrium problem (1.1) under generalized monotonicity conditions in the general setting of FC-spaces without any linear structure.

Lemma 3.1. Let $\left(E,\left\{\phi_{N}\right\}\right)$ be an FC-space and let $K$ be a nonempty closed FC-subspace of $E$. Let $\phi: K \times K \times K \rightarrow \mathbb{R}$ be a real-valued trifunction and let $b: E \times E \rightarrow \mathbb{R}$ be a real-valued bifunction such that

(i) $\phi$ weakly relaxed $\alpha$-monotone, 0 -generalized $R$-diagonally convex and $R$-upper hemicontinuous;

(ii) $b$ is $R$-convex with respect to the second argument;

(iii) $\alpha: E \times E \rightarrow \mathbb{R}$ is $R$-convex with respect to the second argument, with parameter $t^{2}$, i.e.,

$$
\alpha\left(z, x_{t}\right) \leq\left(1-t^{2}\right) \alpha\left(z, x_{1}\right)+t^{2} \alpha\left(z, x_{2}\right)
$$

where $x_{t}=\phi_{N}\left((1-t) e_{0}+t e_{1}\right), t \in[0,1]$ and $N=\left\{x_{1}, x_{2}\right\} \in\langle K\rangle$, and satisfies $\alpha(u, u)=0$ for all $u \in K$.

Then the following problems are equivalent:

$$
\begin{gathered}
\bar{x} \in K, \phi(y, \bar{x}, z)+b(\bar{x}, z)-b(\bar{x}, \bar{x}) \geq 0, \text { for all } y, z \in K, \\
\bar{x} \in K, \phi(y, z, \bar{x})+b(\bar{x}, \bar{x})-b(\bar{x}, z) \leq \alpha(\bar{x}, z), \text { for all } y, z \in K .
\end{gathered}
$$

Proof. Let $\bar{x} \in K$ be a solution of (3.1). Since $\phi$ is weakly relaxed $\alpha$-monotone, we have

$$
\begin{aligned}
\phi(y, z, \bar{x})+b(\bar{x}, \bar{x})-b(\bar{x}, z) & \leq \alpha(\bar{x}, z)-\phi(y, \bar{x}, z)+b(\bar{x}, \bar{x})-b(\bar{x}, z) \\
& \leq \alpha(\bar{x}, z), \text { for all } y, z \in K .
\end{aligned}
$$

So $\bar{x}$ is a solution of (3.2). Conversely, let $\bar{x}$ be a solution of (3.2) and $z$ be an arbitrary element of $K$. Let $N=\{\bar{x}, z\} \in\langle K\rangle$ and

$$
x_{t}=\phi_{N}\left((1-t) e_{0}+t e_{1}\right), t \in(0,1) .
$$

So $x_{t} \in K$. Now replacing $z$ by $x_{t}$ in (3.2), we have,

$$
\phi\left(y, x_{t}, \bar{x}\right) \leq \alpha\left(\bar{x}, x_{t}\right)+b\left(\bar{x}, x_{t}\right)-b(\bar{x}, \bar{x}) .
$$

On the other hand, by using the 0 -generalized $R$-diagonal convexity of $\phi$, we have

$$
t \phi\left(y, x_{t}, z\right)+(1-t) \phi\left(y, x_{t}, \bar{x}\right) \geq 0
$$

Using relation (3.3) and the $R$-convexity of $b(\bar{x}, \cdot)$, we have from realtion (3.4) that

$$
\begin{aligned}
0 & \leq t \phi\left(y, x_{t}, z\right)+(1-t)\left[\alpha\left(\bar{x}, x_{t}\right)+t b(\bar{x}, z)+(1-t) b(\bar{x}, \bar{x})-b(\bar{x}, \bar{x})\right] \\
& \leq t\left[\phi\left(y, x_{t}, z\right)+(1-t) b(\bar{x}, z)-(1-t) b(\bar{x}, \bar{x})+(1-t) \frac{\alpha\left(\bar{x}, x_{t}\right)}{t}\right] .
\end{aligned}
$$


Hence, by using (iii), we deduce

$$
\phi\left(y, w_{t}, v\right)+(1-t) b(\bar{w}, v)-(1-t) b(\bar{w}, \bar{w})+(1-t) t \alpha(\bar{x}, z) \geq 0 .
$$

Since $\phi$ is $R$-upper hemicontinuous, it follows, by considering the upper limit when $t \rightarrow 0^{+}$in (3.5), that

$$
\phi(y, \bar{x}, z)+b(\bar{x}, z)-b(\bar{x}, \bar{x}) \geq 0 .
$$

So $\bar{x}$ satisfies (3.1). This completes the proof.

Theorem 3.1. Let $\left(E,\left\{\phi_{N}\right\}\right)$ be an FC-space and let $K$ be a nonempty closed $F C$-subspace of $E$. Let $\phi$ : $K \times K \times K \rightarrow \mathbb{R}$ be a real-valued trifunction such that $\phi(y, z, z)=0$ for all $y, z \in K$, and let $b: E \times E \rightarrow \mathbb{R}$ be a real-valued bifunction. Suppose that

(i) $\phi$ is weakly relaxed $\alpha$-monotone and $R$-upper hemicontinuous;

(ii) $\phi$ is 0-generalized $R$-diagonally convex and $b$ is $R$-convex with respect to the second argument;

(iii) For each $y \in K$ fixed, the bifunction $\phi_{y}: K \times K \rightarrow \mathbb{R}$ defined by $\phi_{y}(z, x)=\phi(y, z, x)+b(x, x)-$ $b(x, z)-\alpha(x, z)$ is transfer lower semicontinuous with respect to the second argument;

(iv) $\alpha: E \times E \rightarrow \mathbb{R}$ is $R$-convex with respect to the second argument, with parameter $t^{2}$, i.e.,

$$
\alpha\left(z, x_{t}\right) \leq\left(1-t^{2}\right) \alpha\left(z, x_{1}\right)+t^{2} \alpha\left(z, x_{2}\right)
$$

where $x_{t}=\phi_{N}\left((1-t) e_{0}+t e_{1}\right), t \in[0,1]$ and $N=\left\{x_{1}, x_{2}\right\} \in\langle K\rangle$, and satisfies $\alpha(u, u)=0$ for all $u \in K$;

(v) (Coercivity) There exists a nonempty compact subset $D$ of $K$ and $z_{0} \in D, y_{0} \in D$ such that

$$
\phi\left(y_{0}, z_{0}, w\right)+b(w, w)-b\left(w, z_{0}\right)>\alpha\left(w, z_{0}\right), \text { for all } w \in K \backslash D .
$$

Then problem (1.1) has at least one solution.

Proof. Let

$$
A=\left\{y_{1}, y_{2}, \cdots, y_{n}\right\} \in\langle K\rangle
$$

and

$$
\tilde{A}=\left\{y_{1}, y_{2}, \cdots, y_{n}\right\} \cup\left\{y_{0}\right\},
$$

where $y_{0} \in D$ given in the coercivity condition (v). For $z \in K$, let us consider the following set

$$
\mathbb{T}(z)=\bigcap_{y \in \widetilde{A}}\{x \in K: \phi(y, z, x)+b(x, x)-b(x, z) \leq \alpha(x, z)\} .
$$

Note that $\mathbb{T}(z) \neq \emptyset$ since it contains $z$. We claim that $\bigcap_{z \in K} \mathbb{T}(z) \neq \emptyset$. To verify this affirmation, we apply Lemma 2.1. To this aim, first we verify that the family of sets $\{\mathbb{T}(z)\}_{z \in K}$ has the $R$-KKM property. Suppose by contradiction that there exists $N=\left\{z_{0}, z_{1}, \cdots, z_{n}\right\} \in\langle K\rangle$ such that

$$
\phi_{N}\left(\triangle_{n}\right) \nsubseteq \bigcup_{i=0}^{n} \mathbb{T}\left(z_{i}\right) .
$$

Thus, there exists $z_{t}=\phi_{N}\left(\sum_{i=0}^{n} t_{i} e_{i}\right)$ with $t_{i} \geq 0$ for $i=0, \cdots, n$ and $\sum_{i=0}^{n} t_{i}=1$ such that $z_{t} \notin \mathbb{T}\left(z_{i}\right)$ for all $i=0, \cdots, n$. Hence, for each $i \in\{0, \cdots, n\}$, there exists $y_{i} \in \tilde{A}$ such that

$$
\phi\left(y_{i}, z_{i}, z_{t}\right)+b\left(z_{t}, z_{t}\right)-b\left(z_{t}, z_{i}\right)>\alpha\left(z_{t}, z_{i}\right) .
$$

Since $\phi$ is weakly relaxed $\alpha$-monotone, it follows that

$$
\phi\left(y_{i}, z_{t}, z_{i}\right)<b\left(z_{t}, z_{t}\right)-b\left(z_{t}, z_{i}\right), \text { for all } i=0, \cdots, n .
$$


Hence,

$$
\sum_{i=0}^{n} t_{i} \phi\left(y_{i}, z_{t}, z_{i}\right)<b\left(z_{t}, z_{t}\right)-\sum_{i=0}^{n} t_{i} b\left(z_{t}, z_{i}\right) \leq b\left(z_{t}, z_{t}\right)-b\left(z_{t}, z_{t}\right)=0,
$$

which is in contradiction with (ii). On the other hand, from condition (iii) and by taking account of Remark 2.2 and Proposition 2.1, we deduce for each $y \in \tilde{A}$ that

$$
\bigcap_{z \in K}\left\{x \in K: \phi_{y}(z, x) \leq 0\right\}=\bigcap_{z \in K} c l\left(\left\{x \in K: \phi_{y}(z, x) \leq 0\right\}\right),
$$

where $\phi_{y}$ is the real-valued bifunction defined in condition (iii). It follows that

$$
\bigcap_{y \in \tilde{A} z \in K}\left\{x \in K: \phi_{y}(z, x) \leq 0\right\}=\bigcap_{y \in \tilde{A} z \in K} c l\left(\left\{x \in K: \phi_{y}(z, x) \leq 0\right\}\right),
$$

which implies

$$
\bigcap_{z \in K} \mathbb{T}(z)=\bigcap_{z \in K} \bigcap_{y \in \tilde{A}} \operatorname{cl}\left(\left\{x \in K: \phi_{y}(z, x) \leq 0\right\}\right) \supseteq \bigcap_{z \in K} \operatorname{cl}\left(\bigcap_{y \in \tilde{A}}\left\{x \in K: \phi_{y}(z, x) \leq 0\right\}\right) .
$$

As a consequence, we obtain that

$$
\bigcap_{z \in K} \mathbb{T}(z)=\bigcap_{z \in K} \operatorname{cl}(\mathbb{T}(z)) .
$$

Since the family of sets $\{\mathbb{T}(z)\}_{z \in K}$ has the $R$-KKM property, it follows that $\{c l(\mathbb{T}(z))\}_{z \in K}$ has also the $R$-KKM property. Now, let us verify that $c l\left(\mathbb{T}\left(z_{0}\right)\right)$ is a compact set. Indeed, let $w \in \mathbb{T}\left(z_{0}\right)$. Then,

$$
\phi\left(y, z_{0}, w\right)+b(w, w)-b\left(w, z_{0}\right) \leq \alpha\left(w, z_{0}\right), \text { for all } y \in \tilde{A} .
$$

Since $y_{0} \in \tilde{A}$, it follows that

$$
\phi\left(y_{0}, z_{0}, w\right)+b(w, w)-b\left(w, z_{0}\right) \leq \alpha\left(w, z_{0}\right) .
$$

If $w \notin D$, then $w \in K \backslash D$. Hence from the coercivity condition (v), we deduce that

$$
\phi\left(y_{0}, z_{0}, w\right)+b(w, w)-b\left(w, z_{0}\right)>\alpha\left(w, z_{0}\right),
$$

which is in contradiction with (3.7). Therefore, $\mathbb{T}\left(z_{0}\right) \subset D$, which implies that $c l\left(\mathbb{T}\left(z_{0}\right)\right)$ is a compact subset of $E$. Thus, by using Lemma 2.1, we deduce that $\cap_{z \in K} c l(\mathbb{T}(z)) \neq \emptyset$. Consequently, we derive from (3.6) that

$$
\emptyset \neq \bigcap_{z \in K} \mathbb{T}(z) \subset D
$$

Hence, we have proved that, for any $\left\{y_{1}, \cdots, y_{n}\right\} \in\langle K\rangle$, there exists $x \in D$ such that

$$
\phi\left(y_{i}, z, x\right)+b(x, x)-b(x, z) \leq \alpha(x, z), \forall z \in K, \forall i=1, \cdots, n .
$$

For $y \in K$, let us consider the set

$$
\mathbb{U}(y)=\{x \in D: \phi(y, z, x)+b(x, x)-b(x, z) \leq \alpha(x, z), \forall z \in K\} .
$$

From (3.8), we have that $\mathbb{U}(y) \neq \emptyset$ for each $y \in K$, and

$$
\bigcap_{i=1}^{n} \mathbb{U}\left(y_{i}\right) \neq \emptyset \text {, for each }\left\{y_{1}, \cdots, y_{n}\right\} \in\langle K\rangle .
$$

Furthermore, since $U(y)$ can be written as the following

$$
\mathbb{U}(y)=\bigcap_{z \in K}\{x \in D: \phi(y, z, x)+b(x, x)-b(x, z) \leq \alpha(x, z)\},
$$


it follows from assumption (iii) and Proposition 2.1 that

$$
\mathbb{U}(y)=\bigcap_{z \in K} c l(\{x \in D: \phi(y, z, x)+b(x, x)-b(x, z) \leq \alpha(x, z)\}) .
$$

Thus, $\{\mathbb{U}(y)\}_{y \in K}$ is a family of closed subsets of $D$ which has the finite intersection property. Therefore,

$$
\bigcap_{y \in K} \mathbb{U}(y) \neq \emptyset .
$$

This means that there exists $\bar{x} \in D$ such that

$$
\phi(y, z, \bar{x})+b(\bar{x}, \bar{x})-b(\bar{x}, z) \leq \alpha(\bar{x}, z), \forall z \in K, \forall y \in K .
$$

By using Lemma 3.1, we deduce that $\bar{x}$ is a solution of the problem (1.1). This completes the proof.

Remark 3.1. The assumption (iii) of Theorem 3.1 is satisfied if the following three conditions hold:

(1) For each $y, z \in K$, the function $x \in K \times K \mapsto \phi(y, z, x)$ is lower semicontinuous;

(2) $b$ is lower semicontinuous and upper semicontinuous with respect to the first argument;

(3) $\lim \sup \alpha\left(x_{\lambda}, z\right) \leq \alpha(x, z)$ for any net $x_{\lambda}$ converging to $x$ and $z \in K$.

Remark 3.2. In Theorem 3.1, let us suppose that $E$ is a reflexive Banach space endowed with the weak topology $\sigma\left(E, E^{*}\right), K$ is an FC-subspace of $E$ such that $\operatorname{int}(K) \neq \emptyset$ and $\alpha \equiv 0$, i.e., $\phi: K \times K \times K \rightarrow \mathbb{R}$ is monotone. Furthermore, let us assume that $\phi$ is convex and lower semicontinuous with respect to the third argument and that $\phi(y, x, x)=0$ for all $y, x \in K$. Then the coercivity assumption (v) in Theorem 3.1 is satisfied if the following property holds: There exists $z_{0} \in \operatorname{int}(K)$ such that

$$
\frac{b\left(w, z_{0}\right)-b(w, w)}{\left\|w-z_{0}\right\|} \rightarrow-\infty \text { when }\left\|w-z_{0}\right\| \rightarrow+\infty .
$$

Indeed, since $z_{0} \in \operatorname{int}(K)$, there exists $r>0$ such that $K$ contains the closed ball

$$
\bar{B}\left(z_{0}, r\right):=\left\{z \in E:\left\|z-z_{0}\right\| \leq r\right\}
$$

of $E$. We have that $\bar{B}\left(z_{0}, r\right)$ is a convex and $\sigma\left(X, X^{*}\right)$-compact subset of $E$. Since the function $\phi\left(z_{0}, z_{0}, \cdot\right)$ is lower semicontinuous, it follows that there exists $k_{0} \in \mathbb{R}$ such that

$$
\phi\left(z_{0}, z_{0}, x\right)>k_{0}, \text { for all } x \in \bar{B}\left(z_{0}, r\right) .
$$

Let $w \in K \backslash \bar{B}\left(z_{0}, r\right)$ and let us set

$$
\tilde{w}=\frac{r}{\left\|w-z_{0}\right\|} w+\left(1-\frac{r}{\left\|w-z_{0}\right\|}\right) z_{0}
$$

due to $\tilde{w} \in \bar{B}\left(z_{0}, r\right)$. From (3.10), we have that $\phi\left(z_{0}, z_{0}, \tilde{w}\right)>k_{0}$. Since $\phi\left(z_{0}, z_{0}, \cdot\right)$ is a convex function, it follows that

$$
\frac{r}{\left\|w-z_{0}\right\|} \phi\left(z_{0}, z_{0}, w\right)+\left(1-\frac{r}{\left\|w-z_{0}\right\|}\right) \phi\left(z_{0}, z_{0}, z_{0}\right)>k_{0}
$$

Hence,

$$
\phi\left(z_{0}, z_{0}, w\right)>\frac{k_{0}}{r}\left\|w-z_{0}\right\|, \text { for all } w \in K \backslash \bar{B}\left(z_{0}, r\right) .
$$

It follows that

$$
\phi\left(z_{0}, z_{0}, w\right)+b(w, w)-b\left(w, z_{0}\right)>\left\|w-z_{0}\right\|\left[\frac{k_{0}}{r}-\frac{b\left(w, z_{0}\right)-b(w, w)}{\left\|w-z_{0}\right\|}\right], \forall w \in K \backslash \bar{B}\left(z_{0}, r\right) .
$$


From (3.9) and (3.12), we deduce that there exists $\bar{r}>0$ such that

$$
\phi\left(z_{0}, z_{0}, w\right)+b(w, w)-b\left(w, z_{0}\right)>0, \text { for all } w \in K \backslash \bar{B}\left(z_{0}, \bar{r}\right),
$$

which completes the proof of the assertion.

We give the following existence result for problem (1.1) when $\phi$ is relaxed weakly $\alpha$-pseudomonotone and $b \equiv 0$.

Theorem 3.2. Let $\left(E,\left\{\phi_{N}\right\}\right)$ be an FC-space and $K$ be a nonempty closed FC-subspace of $E$. Let $\phi: K \times K \times K \rightarrow \mathbb{R}$ be a real-valued trifunction such that $\phi(y, z, z)=0$ for all $y, z \in K$. Suppose that

(i) $\phi$ is weakly relaxed $\alpha$-pseudomonotone and $R$-upper hemicontinuous;

(ii) $\phi$ is 0-generalized R-diagonally convex;

(iii) For each $y \in K$ fixed, the bifunction $\phi_{y}: K \times K \rightarrow \mathbb{R}$ defined by $\phi_{y}(z, x)=\phi(y, z, x)-\alpha(x, z)$ is transfer lower semicontinuous with respect to the second argument;

(iv) $\alpha: E \times E \rightarrow \mathbb{R}$ is $R$-convex with respect to the second argument, with parameter $t^{2}$, i.e.,

$$
\alpha\left(z, x_{t}\right) \leq\left(1-t^{2}\right) \alpha\left(z, x_{1}\right)+t^{2} \alpha\left(z, x_{2}\right),
$$

where $x_{t}=\phi_{N}\left((1-t) e_{0}+t e_{1}\right), t \in[0,1]$ and $N=\left\{x_{1}, x_{2}\right\} \in\langle K\rangle$, and satisfies $\alpha(u, u)=0$ for all $u \in K$;

(v) (Coercivity) There exists a nonempty compact subset $D$ of $K$ and $z_{0} \in D, y_{0} \in D$ such that

$$
\phi\left(y_{0}, z_{0}, w\right)>\alpha\left(w, z_{0}\right), \text { for all } w \in K \backslash D .
$$

Then, there exists $\bar{x} \in K$ such that

$$
\phi(y, \bar{x}, z) \geq 0, \text { for all } y, z \in K \text {. }
$$

Proof. We follow a similar development to the one used in the proof of Theorem 3.1. For $z \in K$, let us consider the following set

$$
\mathbb{M}(z)=\bigcap_{y \in \widetilde{A}}\{x \in K: \phi(y, z, x) \leq \alpha(x, z)\},
$$

with $\tilde{A}=\left\{y_{1}, y_{2}, \cdots, y_{n}\right\} \cup\left\{y_{0}\right\}$, where $\left\{y_{1}, y_{2}, \cdots, y_{n}\right\}$ is an arbitrary finite subset of $K$ and $y_{0}$ is the element of $K$ considered in the coercivity condition (v). By using the weakly relaxed $\alpha$-pseudomonotonicity of $\phi$ and condition (ii), we verify that the family of sets $\{\mathbb{M}(z)\}_{z \in K}$ has the $R$-KKM property. From condition (iii) and by taking account of Remark 2.2 and Proposition 2.1, we deduce that, for each $y \in \tilde{A}$,

$$
\bigcap_{z \in K}\left\{x \in K: \phi_{y}(z, x) \leq 0\right\}=\bigcap_{z \in K} \operatorname{cl}\left(\left\{x \in K: \phi_{y}(z, x) \leq 0\right\}\right),
$$

where $\phi_{y}$ is the real-valued bifunction defined in condition (iii). It follows that

$$
\bigcap_{y \in \tilde{A} z \in K}\left\{x \in K: \phi_{y}(z, x) \leq 0\right\}=\bigcap_{y \in \tilde{A} z \in K} c l\left(\left\{x \in K: \phi_{y}(z, x) \leq 0\right\}\right),
$$

which implies

$$
\bigcap_{z \in K} \mathbb{M}(z)=\bigcap_{z \in K} \bigcap_{y \in \tilde{A}} c l\left(\left\{x \in K: \phi_{y}(z, x) \leq 0\right\}\right) \supseteq \bigcap_{z \in K} c l\left(\bigcap_{y \in \tilde{A}}\left\{x \in K: \phi_{y}(z, x) \leq 0\right\}\right) .
$$

As a consequence, we obtain that

$$
\bigcap_{z \in K} \mathbb{M}(z)=\bigcap_{z \in K} c l(\mathbb{M}(z)) .
$$


Since the family of sets $\{\mathbb{M}(z)\}_{z \in K}$ has the $R$-KKM property, it follows that $\{c l(\mathbb{M}(z))\}_{z \in K}$ has also the $R$-KKM property. Similarly as in the proof of Theorem 3.1, we have $\operatorname{cl}\left(\mathbb{M}\left(z_{0}\right)\right) \subset D$ and hence it is a compact set. Thus, by using Lemma 2.1, we deduce that $\cap_{z \in K} \operatorname{cl}(\mathbb{M}(z)) \neq \emptyset$. Consequently, we derive from (3.13) that

$$
\emptyset \neq \bigcap_{z \in K} \mathbb{M}(z) \subset D .
$$

Hence, we have proved that for any $\left\{y_{1}, \cdots, y_{n}\right\} \in\langle K\rangle$ there exists $x \in D$ such that

$$
\phi\left(y_{i}, z, x\right) \leq \alpha(x, z), \forall z \in K, \forall i=1, \cdots, n .
$$

For $y \in K$, let us consider the set

$$
\mathbb{V}(y)=\{x \in D: \phi(y, z, x) \leq \alpha(x, z), \forall z \in K\} .
$$

From (3.14) we have that $\mathbb{V}(y) \neq \emptyset$ for each $y \in K$, and

$$
\bigcap_{i=1}^{n} \mathbb{V}\left(y_{i}\right) \neq \emptyset \text {, for each }\left\{y_{1}, \cdots, y_{n}\right\} \in\langle K\rangle .
$$

Furthermore, by using assumption (iii) and Proposition 2.1, we have

$$
\mathbb{V}(y)=\bigcap_{z \in K} \operatorname{cl}(\{x \in D: \phi(y, z, x) \leq \alpha(x, z)\}) .
$$

Thus, $\{\mathbb{V}(y)\}_{y \in K}$ is a family of closed subsets of $D$ which has the finite intersection property. Therefore,

$$
\bigcap_{y \in K} \mathbb{V}(y) \neq \emptyset .
$$

This means that there exists $\bar{x} \in D$ such that

$$
\phi(y, z, \bar{x}) \leq \alpha(\bar{x}, z), \forall z \in K, \forall y \in K .
$$

To conclude, we argue as in the proof of Lemma 3.1.

\section{ApPliCATIONS to MiXed VARIATIONAL-Like INEQUALITIES IN FC-SPACES}

In this section, let $\left(E,\left\{\phi_{N}\right\}\right)$ be an FC-space and $K$ be a nonempty FC-subspace of $E$, where $E$ is a topological vector space. We consider the following nonlinear mixed variational-like inequality problem, in short NMVLIP:

Find $\bar{x} \in K$ such that $\langle N(\bar{x}, y), \eta(z, \bar{x})\rangle+b(\bar{x}, z)-b(\bar{x}, \bar{x}) \geq 0$, for all $y, z \in K$,

where $N: E \times E \rightarrow E^{*}$ is a nonlinear operator with $E^{*}$ as the topological dual of $E, b: E \times E \rightarrow \mathbb{R}$ is a bifunction and $\eta: K \times K \rightarrow E$ is a vector-valued bifunction.

Problem (5.15) was introduced and studied recently by Pany-Mohapatra-Pani [13] in the setting of FC-spaces. Numerous mixed variational inequalities studied earlier can be obtained as special cases from inequality problem $(5.15)$, see, e.g., $[11,16]$ and the references therein.

Theorem 4.1. Let $K$ be a nonempty closed $F C$-subspace of $\left(E,\left\{\phi_{N}\right\}\right)$, where $E$ is a topological vector space with dual space $E^{*}$. Let $N: K \times K \rightarrow E^{*}$ be a nonlinear operator, $b: K \times K \rightarrow \mathbb{R}$ be a real-valued bifunction and $\eta: E \times E \rightarrow E$ be a vector-valued bifunction. Suppose that

(i) $N$ is weakly relaxed $(\eta, \alpha)$-monotone, and $N(y, \cdot)$ is $(\eta, R)$-upper hemicontinuous for each $y \in K$; 
(ii) $N$ is $(\eta, 0)$-generalized $R$-diagonally convex and $b$ is $R$-convex with respect to the second argument;

(iii) For each $y \in K$ fixed, the bifunction $\phi_{y}: K \times K \rightarrow \mathbb{R}$ defined by $\phi_{y}(z, x)=\langle N(z, y), \eta(x, z)\rangle+$ $b(x, x)-b(x, z)-\alpha(x, z)$ is transfer lower semicontinuous with respect to the second argument;

(iv) $\alpha: E \times E \rightarrow \mathbb{R}$ is $R$-convex with respect to the second argument, with parameter $t^{2}$, i.e.,

$$
\alpha\left(z, x_{t}\right) \leq\left(1-t^{2}\right) \alpha\left(z, x_{1}\right)+t^{2} \alpha\left(z, x_{2}\right),
$$

where $x_{t}=\phi_{N}\left((1-t) e_{0}+t e_{1}\right), t \in[0,1]$ and $N=\left\{x_{1}, x_{2}\right\} \in\langle K\rangle$, and satisfies $\alpha(u, u)=0$ for all $u \in K$;

(v) (Coercivity) There exists a nonempty compact subset $D$ of $K$ and $z_{0} \in D, y_{0} \in D$ such that

$$
\left\langle N\left(z_{0}, y_{0}\right), \eta\left(w, z_{0}\right)\right\rangle+b(w, w)-b\left(w, z_{0}\right)>\alpha\left(w, z_{0}\right), \text { for all } w \in K \backslash D .
$$

Then the nonlinear mixed variational-like inequality problem (5.15) has at least one solution.

Proof. It suffices to apply Theorem 3.1 with $\phi(y, x, z)=\langle N(x, y), \eta(z, x)\rangle$.

Remark 4.1. Theorem 4.1 improves Theorem 2 in [13]. Moreover, by the approach developed in this paper, we give a new proof of Theorems 2 and 5 in [13].

\section{APPROXIMATIONS USING THE AUXILIARY PRINCIPLE TECHNIQUE}

In this section, we introduce an iterative method to approximate the solution of the generalized mixed equilibrium problem studied in this paper. It is based on the auxiliary principle technique.

Let $K$ be an FC-subspace of $\left(E,\left\{\phi_{N}\right\}\right)$, where $E$ is a Banach space with dual space $E^{*}$. We consider the following generalized mixed equilibrium problem :

$$
(\mathrm{MEP})\left\{\begin{array}{l}
\text { Find } \bar{x} \in K, \text { such that } \\
\psi(y, \bar{x}, z)+\varphi(\bar{x}, z)-\varphi(\bar{x}, \bar{x}) \geq 0, \text { for all } y, z \in K,
\end{array}\right.
$$

where $\psi: K \times K \times K \rightarrow \mathbb{R}$ is a trifunction and $\varphi: K \times K \rightarrow \mathbb{R}$ is a bifunction.

In order to approximate the solutions of problem (5.1), we consider the following auxiliary problem: For $u \in K$ and $\rho>0$,

$$
\text { (AP) }\left\{\begin{array}{l}
\text { Find } \bar{x} \in K, \text { such that for all } y, z \in K \\
\rho[\psi(y, \bar{x}, z)+\varphi(\bar{x}, z)-\varphi(\bar{x}, \bar{x})]+\langle T(z)-T(\bar{x}), \bar{x}-u\rangle \geq 0
\end{array}\right.
$$

where $T: E \rightarrow E^{*}$ is a given linear operator.

First, we study the existence of solution for auxiliary problem (5.2).

Theorem 5.1. Let $K$ be a nonempty closed $F C$-subspace of $\left(E,\left\{\phi_{N}\right\}\right)$, where $E$ is a Banach space with dual space $E^{*}$. Let $\psi: K \times K \times K \rightarrow \mathbb{R}$ be a real-valued trifunction, $\varphi: E \times E \rightarrow \mathbb{R}$ be a real-valued bifunction and $T: E \rightarrow E^{*}$ be a $\delta$-strongly positive bounded linear operator. Suppose that

(i) $\psi$ is monotone and 0 -generalized $R$-diagonally convex;

(ii) $\varphi$ is skew symmetric, $R$-convex with respect to the second argument;

(iii) $\psi$ is R-upper hemicontinuous and the function $z \in K \mapsto \psi(y, x, z)$ is lower semicontinuous for each fixed $x, y \in K$;

(iv) $\varphi$ is lower semicontinuous and upper semicontinuous with respect to the first argument;

(v) for each fixed $z \in K$, the function $x \in K \mapsto\langle T(x), z\rangle$ is $R$-convex; 
(vi) (Coercivity) for each $u \in K$, there exists a nonempty compact subset $D_{u}$ of $K$ and $z_{0} \in D_{u}, y_{0} \in D_{u}$ such that

$$
\rho\left[\psi\left(y_{0}, z_{0}, w\right)+\varphi(w, w)-\varphi\left(w, z_{0}\right)\right]+\left\langle T(w)-T\left(z_{0}\right), w-u\right\rangle>0, \forall w \in K \backslash D_{u} .
$$

Then for each $u \in K$, the auxiliary problem (5.2) has a unique solution.

Proof. The existence of solution for the auxiliary problem (5.2) follows from Theorem 3.1 by considering the trifunction $\phi: K \times K \times K \rightarrow \mathbb{R}$ and the bifunction $b: E \times E \rightarrow \mathbb{R}$ defined by

$$
\phi(y, x, z)=\rho \psi(y, x, z), \text { and } b(x, z)=\rho[\varphi(x, z)-\varphi(x, x)]+\langle T(z)-T(x), x-u\rangle .
$$

We need only to verify the uniqueness of the solution of the problem (5.2). To this aim, suppose that the problem (5.2) has two solutions $x_{1}$ and $x_{2}$ in $K$. Then, for all $y, z \in K$, we have

$$
\begin{gathered}
\rho\left[\psi\left(y, x_{1}, z\right)+\varphi\left(x_{1}, z\right)-\varphi\left(x_{1}, x_{1}\right)\right]+\left\langle T(z)-T\left(x_{1}\right), x_{1}-u\right\rangle \geq 0, \\
\rho\left[\psi\left(y, x_{2}, z\right)+\varphi\left(x_{2}, z\right)-\varphi\left(x_{2}, x_{2}\right)\right]+\left\langle T(z)-T\left(x_{2}\right), x_{2}-u\right\rangle \geq 0 .
\end{gathered}
$$

By considering $z=x_{2}$ in (5.3) and $z=x_{1}$ in (5.4), and adding the two obtained inequalities, we get

$$
\begin{aligned}
\left\langle T\left(x_{2}-x_{1}\right), x_{2}-x_{1}\right\rangle & \leq \rho\left[\psi\left(y, x_{1}, x_{2}\right)+\psi\left(y, x_{2}, x_{1}\right)\right. \\
& \left.+\varphi\left(x_{2}, x_{1}\right)+\varphi\left(x_{1}, x_{2}\right)-\varphi\left(x_{1}, x_{1}\right)-\varphi\left(x_{2}, x_{2}\right)\right] .
\end{aligned}
$$

Since $\psi$ is monotone, $\varphi$ is skew symmetric and $T$ is $\delta$-strongly positive, it follows that

$$
0 \geq\left\langle T\left(x_{2}-x_{1}\right), x_{2}-x_{1}\right\rangle \geq-\delta\left\|x_{1}-x_{2}\right\|^{2} .
$$

Therefore $x_{1}=x_{2}$, which completes the proof.

Now we give an iterative scheme to approximate the solution of generalized mixed equilibrium problem (5.1).

\section{$\operatorname{Algorithm~(\mathscr {A}):}$}

Step 0: Take $\left.\left\{\rho_{n}\right\}_{n \in \mathbb{N}} \subset\right] 0,+\infty\left[\right.$ such that $\rho_{n} \rightarrow+\infty$, choose $w_{0} \in K$ arbitrary, and let $n:=0$.

Step 1: Given $u_{n} \in K$, compute $u_{n+1} \in K$ such that for all $y, z \in K$,

$$
\rho_{n+1}\left[\psi\left(y, u_{n+1}, z\right)+\varphi\left(u_{n+1}, z\right)-\varphi\left(u_{n+1}, u_{n+1}\right)\right]+\left\langle T(z)-T\left(u_{n+1}\right), u_{n+1}-u_{n}\right\rangle \geq 0 .
$$

Update $n:=n+1$ and go to Step 1 .

In the following theorem, we study the convergence of the algorithm $(\mathscr{A})$.

Theorem 5.2. Let $K$ be a nonempty closed $F C$-subspace of $\left(E,\left\{\phi_{N}\right\}\right)$, where $E$ is a Banach space with dual space $E^{*}$. Let $\psi: K \times K \times K \rightarrow \mathbb{R}$ be a real-valued trifunction, $\varphi: E \times E \rightarrow \mathbb{R}$ be a real-valued bifunction and $T: E \rightarrow E^{*}$ be a $\delta$-strongly positive bounded linear operator. Suppose that

(i) $\psi$ is monotone and 0 -generalized $R$-diagonally convex;

(ii) $\varphi$ is $\gamma$-strongly skew symmetric, $R$-convex with respect to the second argument;

(iii) $\psi$ is R-upper hemicontinuous and the function $z \in K \mapsto \psi(y, x, z)$ is lower semicontinuous for each fixed $x, y \in K$;

(iv) $\varphi$ is lower semicontinuous and upper semicontinuous with respect to the first argument; 
(v) for each fixed $z \in K$, the function $x \in K \mapsto\langle T(x), z\rangle$ is $R$-convex;

(vi) (Coercivity) for each $u \in K$, there exists a nonempty compact subset $D_{u}$ of $K$ and $z_{0} \in D_{u}, y_{0} \in D_{u}$ such that

$$
\rho\left[\psi\left(y_{0}, z_{0}, w\right)+\varphi(w, w)-\varphi\left(w, z_{0}\right)\right]+\left\langle T(w)-T\left(z_{0}\right), w-u\right\rangle>0, \forall w \in K \backslash D_{u} .
$$

Furthermore, we assume that the following condition hold:

$$
\text { (C) } \exists k \in] 0,1\left[\text {, such that } \frac{\rho_{n+1}}{\rho_{n}} \frac{\|T\|}{\left[\delta+\gamma \rho_{n+1}\right]}<k\right. \text {. }
$$

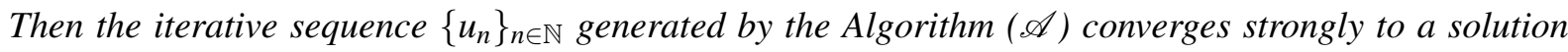
$\bar{u} \in K$ of mixed equilibrium problem (5.1).

Proof. Let us consider the relation (5.5) for $u_{n+1}$ and $u_{n}$. Then, for all $y, z \in K$,

$$
\rho_{n+1}\left[\psi\left(y, u_{n+1}, z\right)+\varphi\left(u_{n+1}, z\right)-\varphi\left(u_{n+1}, u_{n+1}\right)\right]+\left\langle T(z)-T\left(u_{n+1}\right), u_{n+1}-u_{n}\right\rangle \geq 0,
$$

and

$$
\rho_{n}\left[\psi\left(y, u_{n}, z\right)+\varphi\left(u_{n}, z\right)-\varphi\left(u_{n}, u_{n}\right)\right]+\left\langle T(z)-T\left(u_{n}\right), u_{n}-u_{n-1}\right\rangle \geq 0 .
$$

By taking $z=u_{n}$ in relation (5.6) and $z=u_{n+1}$ in relation (5.7), we obtain after dividing by $\rho_{n}$ and $\rho_{n+1}$ and adding the two obtained inequalities

$$
\begin{array}{r}
{\left[\psi\left(y, u_{n+1}, u_{n}\right)+\psi\left(y, u_{n}, u_{n+1}\right)\right]+\left[\varphi\left(u_{n+1}, u_{n}\right)+\varphi\left(u_{n}, u_{n+1}\right)-\varphi\left(u_{n+1}, u_{n+1}\right)\right.} \\
\left.-\varphi\left(u_{n}, u_{n}\right)\right]-\frac{1}{\rho_{n+1}}\left\langle T\left(u_{n+1}-u_{n}\right), u_{n+1}-u_{n}\right\rangle+\frac{1}{\rho_{n}}\left\langle T\left(u_{n+1}-u_{n}\right), u_{n}-u_{n-1}\right\rangle \geq 0 .
\end{array}
$$

Since $\psi$ is monotone and $\varphi$ is $\gamma$-strongly skew-symmetric, it follows from relation (5.8) that

$$
-\gamma\left\|u_{n+1}-u_{n}\right\|^{2}-\frac{1}{\rho_{n+1}}\left\langle T\left(u_{n+1}-u_{n}\right), u_{n+1}-u_{n}\right\rangle+\frac{1}{\rho_{n}}\left\langle T\left(u_{n+1}-u_{n}\right), u_{n}-u_{n-1}\right\rangle \geq 0 .
$$

Hence,

$$
\frac{1}{\rho_{n}}\left\langle T\left(u_{n+1}-u_{n}\right), u_{n}-u_{n-1}\right\rangle \geq \gamma\left\|u_{n+1}-u_{n}\right\|^{2}+\frac{1}{\rho_{n+1}}\left\langle T\left(u_{n+1}-u_{n}\right), u_{n+1}-u_{n}\right\rangle .
$$

Since $T$ is $\delta$-strongly positive and bounded, it follows from (5.10) that

$$
\frac{\|T\|}{\rho_{n}}\left\|u_{n+1}-u_{n}\right\|\left\|u_{n}-u_{n-1}\right\| \geq \gamma\left\|u_{n+1}-u_{n}\right\|^{2}+\frac{\delta}{\rho_{n+1}}\left\|u_{n+1}-u_{n}\right\|^{2} .
$$

Therefore,

$$
\left(\gamma+\frac{\delta}{\rho_{n+1}}\right)\left\|u_{n+1}-u_{n}\right\|^{2} \leq \frac{\|T\|}{\rho_{n}}\left\|u_{n+1}-u_{n}\right\|\left\|u_{n}-u_{n-1}\right\|
$$

This implies

$$
\left\|u_{n+1}-u_{n}\right\| \leq\left(\frac{\rho_{n+1}}{\rho_{n}} \frac{\|T\|}{\left[\delta+\gamma \rho_{n+1}\right]}\right)\left\|u_{n}-u_{n-1}\right\| .
$$

Hence, by taking into account of the condition $(\mathscr{C})$, we deduce

$$
\left\|u_{n+1}-u_{n}\right\| \leq k\left\|u_{n}-u_{n-1}\right\| .
$$

Therefore the sequence $\left\{u_{n}\right\}_{n \in \mathbb{N}} \subset K$ is a Cauchy sequence and hence converges strongly to a point $\bar{u} \in K$. Now, let us verify that $\bar{u}$ is a solution of mixed equilibrium problem (5.1). To this aim, from relation (5.6) we have, for all $y, z \in K$,

$$
\left[\psi\left(y, u_{n+1}, z\right)+\varphi\left(u_{n+1}, z\right)-\varphi\left(u_{n+1}, u_{n+1}\right)\right]+\frac{1}{\rho_{n+1}}\left\langle T(z)-T\left(u_{n+1}\right), u_{n+1}-u_{n}\right\rangle \geq 0 .
$$


Since $\psi$ is monotone, we deduce

$$
\left[\varphi\left(u_{n+1}, z\right)-\varphi\left(u_{n+1}, u_{n+1}\right)\right]+\frac{1}{\rho_{n+1}}\left\langle T(z)-T\left(u_{n+1}\right), u_{n+1}-u_{n}\right\rangle \geq \psi\left(y, z, u_{n+1}\right) .
$$

By considering the lower limit when $n \rightarrow+\infty$ in the previous inequality, and taking account of the conditions (iii) and (iv), we obtain

$$
\varphi(\bar{u}, z)-\varphi(\bar{u}, \bar{u}) \geq \psi(y, z, \bar{u}) \text { for all } y, z \in K .
$$

We complete the proof by using Lemma 3.1 with $\phi=\psi, b(x, z)=\varphi(x, z)-\varphi(x, x)$ and $\alpha \equiv 0$.

Remark 5.1. The condition $(\mathscr{C})$ is satisfied if we consider for instance $\rho_{n}=n^{p}$ with $p>1$. The algorithm $(\mathscr{A})$ is as fast as $p$ takes high values.

Now, as an application, we study the approximation of solutions for the following nonlinear mixed variational-like inequality: Find $x \in K$ such that

$$
\langle N(x, y), \eta(z, z)\rangle+b(x, z)-b(x, x) \geq 0, \text { for all } y, z \in K,
$$

where $K$ be a nonempty closed FC-subspace of $\left(E,\left\{\phi_{N}\right\}\right), E$ is a Banach space with dual space $E^{*}$, $N: E \times E \rightarrow E^{*}$ is a nonlinear operator, $b: E \times E \rightarrow \mathbb{R}$ is a bifunction and $\eta: K \times K \rightarrow E$.

The following algorithm permits to have an approximation of the solution for the nonlinear mixed variational-like inequality (5.15).

\section{$\operatorname{Algorithm}(\mathscr{B})$ :}

Step 0: Take $\left.\left\{\rho_{n}\right\}_{n \in \mathbb{N}} \subset\right] 0,+\infty\left[\right.$ such that $\rho_{n} \rightarrow+\infty$, choose $w_{0} \in K$ arbitrary, and let $n:=0$.

Step 1: Given $u_{n} \in K$ compute $u_{n+1} \in K$ such that for all $y, z \in K$,

$$
\begin{array}{r}
\rho_{n+1}\left[\left\langle N\left(y, u_{n+1}\right), \eta\left(z, u_{n+1}\right)\right\rangle+\varphi\left(u_{n+1}, z\right)-\varphi\left(u_{n+1}, u_{n+1}\right)\right] \\
+\left\langle T(z)-T\left(u_{n+1}\right), u_{n+1}-u_{n}\right\rangle \geq 0 .
\end{array}
$$

Update $n:=n+1$ and go to Step 1 .

Here $T: E \rightarrow E^{*}$ is a $\delta$-strongly positive linear and bounded operator.

The convergence of the algorithm $(\mathscr{B})$ is analyzed in the following corollary, which is a direct consequence of Theorem 5.2.

Corollary 5.1. Let $K$ be a nonempty closed $F C$-subspace of $\left(E,\left\{\phi_{N}\right\}\right)$, where $E$ is a Banach space with dual space $E^{*}$. Let $N: K \times K \rightarrow E^{*}$ be a nonlinear operator, $\varphi: E \times E \rightarrow \mathbb{R}$ be a real-valued bifunction, $\eta: E \times E \rightarrow E$ be a functional such that $\eta(u, v)=-\eta(v, u)$ for all $u, v \in E$, and $T: E \rightarrow E^{*}$ be a $\delta$-strongly positive bounded linear operator. Suppose that

(i) $N$ is $\eta$-monotone and $(\eta, 0)$-generalized $R$-diagonally convex;

(ii) $\varphi$ is $\gamma$-strongly skew symmetric and $R$-convex with respect to the second argument;

(iii) $N(\cdot, y)$ is $(\eta, R)$-upper hemicontinuous, and the function $z \in K \times K \mapsto\langle N(x, y), \eta(z, x)\rangle$ is lower semicontinuous for each fixed $(x, y) \in K \times K$;

(iv) $\varphi$ is lower semicontinuous and upper semicontinuous with respect to the first argument;

(v) for each fixed $z \in K$, the function $x \in K \mapsto\langle T(x), z\rangle$ is $R$-convex; 
(vi) (Coercivity) for each $u \in K$, there exists a nonempty compact subset $D_{u}$ of $K$ and $z_{0} \in D_{u}, y_{0} \in D_{u}$ such that

$$
\rho\left[\left\langle N\left(z_{0}, y_{0}\right), \eta\left(w, z_{0}\right)\right\rangle+\varphi(w, w)-\varphi\left(w, z_{0}\right)\right]+\left\langle T(w)-T\left(z_{0}\right), w-u\right\rangle>0, \forall w \in K \backslash D_{u} .
$$

Furthermore, we assume that the following condition hold:

$$
\text { (C) } \exists k \in] 0,1\left[\text {, such that } \frac{\rho_{n+1}}{\rho_{n}} \frac{\|T\|}{\left[\delta+\gamma \rho_{n+1}\right]}<k .\right.
$$

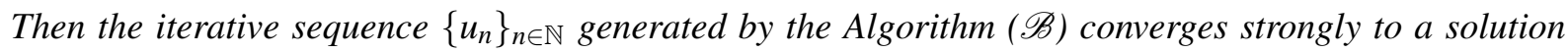
$\bar{u} \in K$ of nonlinear mixed variational-like inequality problem (5.15).

\section{CONCLUSION}

In this paper, we studied the existence and approximation of the solution of the mixed equilibrium problem with a trifunction. The main results are established by relaxing the convexity structure and the linearity condition on the space by using the R-KKM principle in the framework of generalized monotonicity, which is the weakly relaxed $\alpha$-monotonicity and the weakly relaxed $\alpha$-pseudomonotonicity. The approximation of solutions for problem (1.1), where $K$ is an FC-subspace of a Banach space $E$ is studied by means of the auxiliary principle technique. We give an algorithm which converges strongly to a solution of problem (1.1), and an application to the approximation of solutions of the mixed variationallike inequalities in FC-spaces is given. The beauty of the problem considered in this paper is that it encompasses several important type of problems as special cases. The approach developed in this paper generalizes and improve the recent results obtained in $[13,18]$.

\section{Acknowledgments}

The second and third authors are grateful to the Mohapatra Family Foundation and the College of Graduate Studies of the University of Central Florida for their support.

\section{REFERENCES}

[1] A.S. Antipin, The Fixed points of extremal maps: computation by gradient methods, Zh. Vychisl. Mat. Mat. Fiz. 37 (1997), 42-53.

[2] M.R. Bai, S.Z. Zhou, G.Y. Ni, Variational-like inequalities with relaxed $\eta-\alpha$ pseudomonotone mappings in Banach spaces, Appl. Math. Lett. 19 (2006), 547-554.

[3] H. Ben-El-Mechaiekh, S. Chebbi, M. Florenzano, J.V. Llinares, Abstract convexity and fixed points, J. Math. Anal. Appl. 222 (1998), 138-150.

[4] E. Blum, W. Oettli, From optimization and variational inequalities to equilibrium problems, Mathematics Stud. 63 (1994), 123-145.

[5] Q. Chen, J. Luo, Relaxed $\eta-\alpha$ quasimonotone and application to the generalized variational-like inequality problem, J. Inequal. Appl. 1 (2013), 1-8.

[6] X.P. Ding, System of coincidence theorems in product topological spaces and applications, Appl. Math. Mech. 26 (2005), 1547-1555.

[7] X.P. Ding, Maximal element theorems in product FC-spaces and generalized games, J. Math. Anal. Appl. 305 (2005), 29-42.

[8] X.P. Ding, New H-KKM theorems and equilibria of generalized games, Indian J. Pure Appl. Math. 27 (1996), $1057-1071$.

[9] L. Deng, X. Xia, Generalized R-KKM theorem in topological space and their applications, J. Math. Anal. Appl. 285 (2003), 679-690.

[10] C.D. Horvath, Contractibility and general convexity, J. Math. Anal. Appl. 156 (1991), 341-357. 
[11] N.J. Huang, C.X. Deng, Auxiliary principle and iterative algorithms for generalized set-valued strongly nonlinear mixed variational-like inequalities, J. Math. Anal. Appl. 256 (2001), 345-359.

[12] Z. Liu, S. Zeng, Equilibrium problems with generalized monotone mapping and its applications, Math. Methods Appl. Sci. 39 (2016), 152-163.

[13] G. Pany, R.N. Mohapatra, S. Pani, Solution of a class of equilibrium problems and variational inequalities in FC-spaces, Ann. Oper. Res. 269 (2018), 565-582.

[14] G. Pany, S. Pani, Nonlinear mixed variational-like inequality with respect to weakly relaxed $\eta$ - $\alpha$ monotone mapping in Banach spaces, In: P.N. Agrawal, R. Mohapatra, U. Singh, H.M. Srivastava, (Eds.) Mathematical Analysis and its Applications, vol. 143, pp. 185-196, Roorkee, India, 2015.

[15] S. Park, H. Kim, Foundations of the KKM theory on generalized convex spaces, J. Math. Anal. Appl. 209 (1997), $551-571$.

[16] M.V. Solodov, Merit functions and error bounds for generalized variational inequalities, J. Math. Anal. Appl. 287 (2003), 405-414.

[17] G. Q. Tian, Generalizations of the FKKM Theorem and the Ky Fan Minimax Inequality, with Applications to Maximal Elements, Price Equilibrium, and Complementarity, J. Math. Anal. Appl. 170 (1992), 457-471.

[18] J. Wang, The existence of solutions for general variational inequality and applications in FC-spaces, J. Inequal. Appl. 2012 (2012), Article ID 49. 